\title{
Ostarrichislam. Gründe der korporatistischen Hereinnahme des Islams in der Zweiten Republik
}

\author{
Farid Hafez ${ }^{1, *}$ \\ 1 Universität Salzburg, Abteilung Politikwissenschaft, Salzburg, Österreich \\ * E-Mail: farid.hafez@sbg.ac.at
}

\section{Zusammenfassung}

Österreich ist als eines der wenigen westeuropäischen Länder bekannt für seine korporatistische Hereinnahme des Islams. Die ,liberale Islampolitik spiegelt sich etwa in der Tatsache wider, dass es in Österreich im Unterschied zu Deutschland, Belgien oder Frankreich zu keinem Kopftuchverbot gekommen ist. Bei der Begründung dieser, liberalen` Politik wird in der rechtswissenschaftlichen und politikwissenschaftlichen Literatur meist auf die gesetzliche Anerkennung des Islams im historischen Islamgesetz von 1912 verwiesen. Dieser Artikel testet zwei Theorien, warum es 1979 zu einer Genehmigung der Islamischen Glaubensgemeinschaft in Österreich als Körperschaft öffentlichen Rechts neben heute I5 anderen Kirchen und Religionsgesellschaften gekommen ist. Neben dem historischen Institutionalismus, der aufgrund der spezifisch historisch gewachsenen Staat-Kirche-Beziehungen im Zusammenhang mit dem Islam von Bedeutung zu sein scheint, wird die Theorie Politischer Möglichkeitsstrukturen zur Erklärung herangezogen. Der Artikel argumentiert, dass die Theorie der Politischen Möglichkeitsstrukturen erklärt, warum es 1979 zu einer Anerkennung kam, während die Theorie des historischen Institutionalismus erklärt, welcher Form diese Anerkennung war.

\section{Schlüsselwörter}

Historischer Institutionalismus, Politische Möglichkeitsstrukturen, Kirche, Islam, Österreich, Islamgesetz

\section{Austrian Islam. Explaining the Corporatist Inclusion of Islam in the Second Republic of Austria}

\begin{abstract}
Austria is one of the very few Western European countries that has incorporated Islam in its polity. This laid the basis for a "liberal Islam-Politics' as can be seen with the fact that there are no bans for the hijab such as is the case in Germany, Belgium or France. In political science and legal studies literature, this is mainly explained by referring to the legal recognition of Islam by the I9I2 Islam bill. The assumption that path dependency explains the nature of incorporation of Islam into the political system of Austria, is standing to reason. This article shows that the theory of historical institutionalism cannot explain why the Islamic Council was recognized in 1979 as a corporate body, representing today one of I6 legally recognized churches and religious communities. I argue that the theory of political opportunity structures helps explaining, why the Islamic Council was recognized in 1979 .
\end{abstract}

\section{Keywords}

Historical Institutionalism, Political Opportunity Structures, Church, Islam, Austria, Islam-law

The author has declared that no competing interests exist. 


\section{Einleitung}

Österreich ist ein Land mit einem ethno-kulturellen ,Citizenship Regime؛. Im Gegensatz zu Ländern mit multikultureller oder republikanischer Ausrichtung, die Menschen meist einen leichteren Zugang zur Staatsbürgerschaft verschaffen und damit die Grenzziehungen, wer zur Nation gehört und wer nicht, weniger restriktiv handhaben, gilt Österreich als Land mit restriktiven Staatsbürgerschaftsgesetzen (Bauböck/Perchinig 2006) und einer tendenziell homogenen Vorstellung österreichischer Identität (Wodak/ De Chilla/Reisigl I998; Krzyzanowski/Wodak 20II). Im Zusammenhang mit dem Islam als Minderheitenreligion ist speziell anzumerken, dass in Österreich die Katholische Kirche eine dominante Rolle inne hat (Hanisch 1977, 1994), was auch vielerlei Privilegien im Gegensatz zu anderen gesetzlich anerkannten Kirchen und Religionsgesellschaften (KuR) inkludiert (Kalb/Schinkele/Potz 2003, 446-540). Weiters ist die langjährige diskriminierende Behandlung von Volksgruppen-Minderheiten ebenso ein Merkmal österreichischer Minderheitenpolitik (Reiterer 1996). Hinzu kommt eine zentrale Rolle des Antisemitismus, wie er im Nationalsozialistischen Regime zur industriellen Vernichtung von Jüdinnen und Juden geführt hat, und in Form des sekundären Antisemitismus nicht als vollständig überwunden betrachtet werden kann, wie die Affären um Bruno Kreisky (Pelinka/Sickinger/Stögner 2008), Kurt Waldheim (Gruber I99I) oder in jüngerer Zeit um Ariel Muzicant verdeutlichen (Pelinka/Wodak 2002). Insbesondere ist für die Imagination des Islams in Österreich zu sagen, dass eine spezifische Ausformung des Orientalismus, wie ihn der Anthropologe André Gingrich herausgearbeitet hat (Gringrich 2015) den Islam mitunter als Narrativ der Bedrohung in die Alltagskultur eingeschrieben hat. Jedoch war im Umgang mit dem Islam vor der aktiven Bemächtigung islamophober Wahlkampfstrategien durch die FPÖ im Jahre 2005 (Hafez 20IO; 2015, Hödl 20IO) ein inklusiver Umgang staatlicher Behörden mit dem Islam beobachtbar, was sich insbesondere in der gesetzlichen Anerkennung des Islams durch das Islamgesetz aus dem Jahre I9I2 und der darauf basierenden Genehmigung der Islamischen Glaubensgemeinschaft als Religionsgesellschaft und damit Körperschaft öffentlichen Rechts (Kalb/Schinkele/Potz 2003) sowie dem einhergehenden inklusiven Islamdiskurs der politischen Eliten zeigt (Hafez 2OI4). Wie aber lässt sich diese inklusive Hereinnahme vor diesem Hintergrund erklären?

Vergleicht man Österreich mit verschiedenen anderen europäischen Ländern und vergegenwärtigt man sich etwa die restriktiven Kopftuchpolitiken in Ländern wie Deutschland, Belgien oder Frankreich (Berghahn/Rostock 2009) oder die Nichtanerkennung des Islams in Deutschland, die im Vergleich zu Österreich bis vor kurzem noch eine Institutionalisierung Islamischer Theologie an Universitäten oder aber eine Einrichtung des islamischen Religionsun- terrichts an öffentlichen Schulen in den meisten Ländern nicht möglich machte (Spielhaus/Herzog 2015), so ist festzuhalten, dass Österreich in bestimmten Policy-Bereichen wie etwa in der Kopftuchpolitik (Gresch et al. 2008, 2012) eine ,liberale' Islampolitik zugeschrieben wird. Auch andere Arbeiten streichen die österreichische Islampolitik als offen hervor und verweisen auf die rechtliche Anerkennung zur Erklärung, wie über den Islam in Österreich im Gegensatz $\mathrm{zu}$ anderen Ländern in der Öffentlichkeit debattiert wird (Dolezal/Helbling/Hutter 20IO). Jedoch wird diese ,liberale“ Islampolitik in diesen Arbeiten vorausgesetzt bzw. bestenfalls mit Verweis auf die rechtliche Anerkennung des Islams mit dem IslamG I9I 2 erklärt (Gresch et al. 2008, 420). So etwa Permoser und Rosenberger, die die korporatistische Hereinnahme des Islams mit der rechtlichen Anerkennung des Islams in Österreich, wie sie in der Habsburger Monarchie im Jahre 19I2 folgend auf die Annexion von Bosnien und Herzegowinas im Jahre 1909 geschah, erklären. Sie argumentieren, dass die gesetzliche Anerkennung „nicht das Produkt einer multikulturellen Politikauffassung hinsichtlich ethnischer und religiöser Diversität, sondern die Folge historisch-politischer Pfadabhängigkeiten" sei (Permoser/Rosenberger 20I2, 74). Permoser und Rosenberger können sich in dieser Argumentation theoretisch auf eine fundierte vergleichende Analyse der Akkomodation islamischer Religion in Großbritannien, Frankreich und Deutschland der beiden Politikwissenschaftler Fetzer und Soper (2005) berufen, die besagt, dass die Islampolitik in westeuropäischen Staaten zentral von den vorhandenen Staats-Kirche-Beziehungen beeinflusst wird, womit im Wesentlichen historisch-institutionalistisch argumentiert wird. Empirisch stützen sie sich auf rechtswissenschaftliche Arbeiten. Hier meine ich, dass es eine Lücke in der rechtswissenschaftlichen wie auch politikwissenschaftlichen Literatur, die die Genehmigung der Islamischen Glaubensgemeinschaft in Österreich (IGGiÖ) im Jahre 1979 basierend auf dem IslamG I9I2 ohne weiterem Nachfragen als gegeben annimmt. Die Empirie zeigt, dass im Gegensatz zu anderen Kirchen und Religionsgesellschaft die Islamische Glaubensgemeinschaft in Österreich nur zögerlich Genehmigung auf Basis des IslamG 1912 erhalten hat (siehe Kapitel 3 und 4 in diesem Artikel). Genau aber diese Prämisse in der rechtswissenschaftlichen wie politikwissenschaftlichen Literatur, wonach der Verweis auf die rechtlichen Grundlagen des IslamG I9I2 zur Erklärung, warum es zur Anerkennung der IGGiÖ gekommen sei, genüge, wird hier hinterfragt. Es wird der Frage nachgegangen, warum es 1979 zur Genehmigung einer islamischen Religionsgesellschaft, der IGGiÖ, kam. Präziser: Genügt der Verweis auf die rechtliche Anerkennung des Islams 
im Jahre I9I2? Wenn nicht, welche Theorien können die rechtliche Inklusion des Islams in Österreich im Jahre I979 erklären?

Dazu werden zwei Theorien getestet: I) der historische Institutionalismus mithilfe des Konzeptes der Pfadabhängigkeit, welches bisweilen zur Erklärung genannt wurde, eine systematische Überprüfung bisher aber vermissen lässt. 2) die Theorie der politischen Möglichkeitsstrukturen, welche im Gegensatz zur Pfadabhängigkeit externe Faktoren zur Erklärung politischer Handlungen heranzieht. Dieser Artikel beabsichtigt eine Ergründung, warum es 1979 zu einer korporatistischen Hereinnahme des Islams kam und nicht in welcher Form. Denn das wie erklärt sich vermutlich durchaus mit einem Verweis auf das österreichische Religionsrecht und der Theorie der Pfadabhängigkeit, wenn auch - wie gerade gesagt - eine systematische Überprüfung der Islampolitik seit 1979 bisher ausgeblieben ist. Damit will dieser Artikel die nicht weiter überprüfte Annahme, wie sie etwa von Permoser und Rosenberger (2012) gemacht wird, testen, wonach Pfadabhängigkeit die liberale Islampolitik erkläre. Sie berufen sich in ihrer Annahme im Wesentlichen auf rechtswissenschaftliche Arbeiten, wie es für die Politikwissenschaft lange Jahre im Hinblick auf die Untersuchung von Religionspolitik in Österreich der Fall war. Das zeigt etwa auch Barbara Prainsacks Artikel über Religion und Politik in der letzten Ausgabe des Handbuchs „Politik in Österreich", der sich beinahe ausschließlich auf rechtswissenschaftliche Literatur beruft (Prainsack 2006). Da die Politikwissenschaft aber mehr eine hinterfragende als eine legalistische und dabei oftmals deskriptive Perspektive einnimmt, wie es für weite Teile der Rechtswissenschaften gilt, ist dieser Beitrag auch als eine politikwissenschaftliche Intervention in ein bisher beinahe vollständig den Rechtswissenschaften überlassenes Forschungsfeld zu lesen.

Zur Überprüfung der Theorie zur Erklärung der korporatistischen Hereinnahme des Islams wird methodisch auf das ,process tracing" - „das Aufspüren oder Nachverfolgen eines bestimmten Vorganges" (Muno 2009, I25) - zurückgegriffen. Process tracing wurde 1979 erstmals entwickelt und 2005 durch Bennett und George (2005) umfassend theoretisiert. Process tracing ist eine offene Methode, die meist bei der Untersuchung von Einzelfällen aber auch vergleichend angewandt wird. Sie erlaubt die Überprüfung von Hypothesen, identifiziert Kausalmechanismen, die unabhängige und abhängige Variablen miteinander verbindet (Collier 2OII, 823). Während manche AutorInnen einen Nachteil darin sehen, dass diese Methode der Rekonstruktion linearer Zusammenhänge dient (und damit, so die Kritik, komplexe Zusammenhänge nicht in den Blick nimmt) (Muno 2009, I25), erscheint dies gerade im Zusammenhang mit dem historischen Institutionalismus von Vorteil zu sein. Ausführliche Beschreibungen von aufeinander folgen- den Sequenzen und gleichzeitig das Festhalten einzelner Momente werden als grundlegend für das ,process tracing' beschrieben (Collier 20II, 824). Zur Analyse dieser historischen Sequenzen werden insbesondere die Schreiben von Ministerien herangezogen. Zusätzlich wird auch der öffentliche Diskurs um den Islam, sowie öffentliche Auftritte von RegierungsvertreterInnen, Interviews in Zeitungen, etc. im genannten Zeitraum bzw. aus der Retrospektive herangezogen. Darüber hinaus wurden ExpertInneninterviews gemacht, um Einblicke in einzelne Sequenzen zu erhalten, die lange zurückliegen, wenn auch viele der zentralen AkteurInnen bereits verstorben sind, was eine minutiöse Deskription unmöglich macht. Insofern operiert mein Text nicht auf der Ebene eines "doubly decisive test", wonach Hypothesen bestätigt werden und gleichzeitig eine Vielzahl anderer Hypothesen eliminiert wird, sondern auf der Ebene einer darunter liegenden, „smoking-gun“-Beweisführung, welche eine Hypothese bestätigt, ohne jedoch eine Vielzahl anderer Hypothesen vollständig ausschließen zu können. Dies begründet sich insbesondere vor dem Hintergrund des langen Zeitraums, der Tatsache, dass handelnde AkteurInnen aus dieser Zeit nicht mehr leben und daher nicht mehr befragt werden können.

Im nächsten Kapitel werden der historische Institutionalismus (Pfadabhängigkeit) sowie politische Möglichkeitsstrukturen als zwei Theorien vorgestellt, um in den zwei darauffolgenden Kapiteln ( 3 und 4) die beiden Theorien an der Empirie zu testen. Im abschließenden Kapitel werden die Ergebnisse zusammengefasst und dargestellt.

\section{Historischer Institutionalismus und Politische Möglichkeitsstrukturen}

\subsection{Historischer Institutionalismus}

Der historische Institutionalismus als eine Spielart von - je nach Kategorisierung - drei bis fünf Versionen ist eher dem Strukturalismus verbunden und geht davon aus, dass Institutionen die primären Faktoren für kollektives Handeln, Politikergebnisse, aber auch Identitäten, Interessen und Interaktionen sind. Damit sind sie gleichzeitig erklärende Variablen (Hall/Taylor 1996, 937). Vier zentrale Merkmale machen Hall und Taylor für diesen Ansatz aus: I. Historischer Institutionalismus würde das Verhältnis von Institutionen und individuellem Verhalten sehr breit konzeptualisieren. 2. Der historische Institutionalismus betont die Machtasymmetrien bei der Bildung und Entwicklung von Institutionen. 3. Die Entwicklung von Institutionen werden durch Pfadabhängigkeit erklärt. 4. Der Faktor ,Ideen' wird in die Analyse von Institutionen hineingenommen (ebd., 94If.). Nach Guy Peters unterscheidet den historischen 
Institutionalismus von anderen institutionalistischen Theorien das Postulat ,time matters', also der Faktor der historischen Dimension (Peters 1999, 63). Demnach würden Entscheidungen politischer AkteurInnen zu einem Zeitpunkt zeitlich spätere Entscheidungen in dem gleichen Politikfeld prägen (ebd. 63ff.). Damit fokussiert historischer Institutionalismus auf die Analyse von Kontinuitäten, kann aber ebenso Brüche erklären, wenn dies auch von KritikerInnen oftmals angezweifelt wird (Peters/Pierre/King 2005).

Eine zentrale Kategorie des historischen Institutionalismus bildet der Begriff der ,Pfadabhängigkeit' (path dependency), der von Stephen Krasner (1984) geliehen wurde. Pfadabhängigkeit besagt, dass politische Entscheidungen die gesamte Entwicklung in einem Politikfeld nachhaltig beeinflussen. Was in der Vergangenheit politisch praktiziert wurde, so die Annahme, wirke sich auf die politische Praxis der Gegenwart aus und bestimme den Entwicklungspfad in dem entsprechenden Politikbereich. Dieser Pfad kann zwar verlassen werden, dafür seien aber stärkere Impulse wie etwa ein militärischer Konflikt notwendig (Hall/Taylor 1996, 942). Da Revolutionen und andere exogene Faktoren nicht zum politischen Alltag gehören, stellt ein Pfadwechsel eher die Ausnahme dar (Kritik daran: Beyer 2006). Das bedeutet aber nicht, dass die These der Pfadabhängigkeit keinen Wandel kennen würde. Im Gegenteil liegt die Aufmerksamkeit des historischen Institutionalismus auf Fragen des Wandels und der Kontinuität von Institutionen (Helms 2004, 28).

Mithilfe des historischen Institutionalismus müsste also argumentiert werden, dass Handlungen im Feld der österreichischen Religionspolitik vor 1979 eine Entscheidung im Hinblick auf die mögliche Anerkennung einer islamischen Religionsgesellschaft prägen würden.

\subsection{Politische Möglichkeitsstrukturen}

Der innovative Ansatz dieses Artikels liegt darin, zu klären, warum es im Jahre 1979 zu einer Anerkennung einer islamischen Religionsgesellschaft kam. Hierfür werden exogene Faktoren mithilfe der Theorie politischer Möglichkeitsstrukturen (political opportunity structure) zur Erklärung herangezogen. Der Begriff der Politischen Möglichkeitsstrukturen wird in der Literatur sehr unterschiedlich verwendet, sowohl was die Definition wie auch die Operationalisierbarkeit des Konzeptes anbelangt (Goodwin/Jasper 1999, 3Iff.). Jon Elster versteht unter dem opportunity set die Handlungsmöglichkeiten innerhalb eines bestimmten Kontextes, aus denen AkteurInnen entsprechend ihrer Präferenzen wählen können (Elster 1992, I3). Doug McAdam und andere haben eine Konzeptualisierung vorgenommen, um einer Schwammigkeit des Konzeptes entgegenzutreten. So meint McAdam, dass die Frage der Offenheit vs. Ge- schlossenheit eines politischen Systems ein zentrales Charakteristikum darstelle. Weiters gäbe die Haltung und Kohärenz der Eliten, die Frage des Vorhandenseins oder Fehlens von Verbündeten sowie die Fähigkeit des Staates, repressive Maßnahmen bereitzustellen, Auskunft über die politischen Möglichkeitsstrukturen innerhalb eines politischen Systems (McAdam I996, 26). In diesem Artikel werden politische Möglichkeitsstrukturen nicht im Sinne von strukturellen Veränderungen innerhalb des politischen Systems konzeptionalisiert, sondern als Themen-spezifische Möglichkeitsfenster verstanden (Meyer/Minkoff 2004). Mit dem Begriff des Möglichkeitsfensters (windows of opportunity) wird angesprochen, dass aufgrund von strukturellen Auslösern bestimmte Ereignisse auf die politische Agenda kommen, die wiederum auf struktureller Ebene neue Politiken ermöglichen. John Kingdon hat dies als policy window bezeichnet:

„The policy window is an opportunity for advocates of proposals to push their pet solutions, or to push attention to their special problems. [...] These policy windows, opportunities for action on given initiatives, present themselves and stay open for only short periods. If participants cannot or do not take advantage of these opportunities, they must bide their time until the next opportunity comes along " (Kingdon 1984, I73f.).

So schnell wie Themen auf die Agenda gesetzt werden können, können sie auch wieder verschwinden. Für AkteurInnen ist es deswegen zentral, diese Möglichkeitsfenster identifizieren zu können, um sie zu nutzen. Beyer knüpft an Lipset und Rokkan an, wenn er das Konzept des window of oportunity in die These der Pfadabhängigkeit integriert und damit jenen Zeitpunkt benennt, zu dem ein neuer Pfad eingeschlagen werden kann, weil verschiedenen Optionen zur Verfügung stehen (Beyer 2006, 26). Insofern scheint es angemessen, die Theorie der politischen Möglichkeitsstrukturen in dem vorliegenden Untersuchungsfall genau hier zu verorten.

Weil die inklusive, korporatistische Hereinnahme des Islams nicht alleine mit dem Verweis auf das IslamG I9I2 und die Pfadabhängigkeit religionspolitischen Handelns erklärt werden kann, wird angenommen, dass es politischer Möglichkeitsstrukturen bedurfte, um diese Pfadabhängigkeit zu revitalisieren. Denn zum einen war das IslamG 1912 von 1912 in der Habsburger Monarchie über die Erste Republik, den Austrofaschismus wie auch dem Nationalsozialismus bis in die Zweite Republik bis zur Anerkennung der IGGiÖ im Jahre I979 de facto ein totes Gesetz. Zum anderen zog sich der Prozess um Anerkennung als gesetzlich eingetragene Religionsgesellschaft ganze zehn Jahre ab 1969. 


\section{Pfadabhängigkeit in der Islampolitik}

Nachdem das Recht auf Religionsfreiheit im privaten Bereich, wie es im Josephinischen Toleranzpatent von I78I festgeschrieben wurde (Wandruszka I985, xivf.), auf das öffentliche Recht auf Religionsausübung im Anerkennungsgesetz von 1874 ausgeweitet wurde, kam es in der Folge nicht nur zur gesetzlichen Anerkennung von anderen Kirchen und Religionsgesellschaften neben der Katholischen Kirche, sondern auch zu sogenannten Religionssondergesetzen. Neben dem ProtestantenG I867und dem IsraelitenG I89o betraf dies auch die MuslimInnen, nachdem 1909 Bosnien und Herzegowina von der Habsburger Monarchie annektiert wurden (Wandruszka 1985, xvf.). Drei Jahre später kam es zur gesetzlichen Anerkennung des Islams, was nicht nur der Pluralismusfähigkeit der Doppelmonarchie geschuldet war, sondern einem kolonialen Herrschaftsprojekt, das die bosnischen MuslimInnen von der Nabelschnur des Osmanischen Reiches abzutrennen versuchte und es gleichzeitig stärker an die Krone binden wollte (Furat 2012). Das IslamG I9I2 ging davon aus, dass es weniger um eine Anerkennung gemäß dem an christlichen Organisationsformen orientierten allgemeinen Anerkennungsrecht ging, denn um eine gleichgestellte Anerkennung der AnhängerInnen der islamischen Religion mit den Angehörigen anderer gesetzlich anerkannter Kirchen und Religionsgesellschaften (Potz 20I3, 48). Aufgrund des Ausbruchs des Ersten Weltkrieges und der niedrigen Anzahl an MuslimInnen in der Ersten Republik kam es vorerst zu keiner Ordnung der äußeren Rechtsverhältnisse, wie es das Gesetz von den MuslimInnen verlangte. Es wurde also keine Religionsgesellschaft gegründet. Tatsächlich sind mit dem Ende des Ersten Weltkrieges nicht nur die muslimischen Bosniaken als muslimische Bevölkerung Österreichs weggefallen. Die Anzahl an MuslimInnen verringerte sich in der Zwischenkriegszeit auf etwa Iooo Personen (in Wien) (Strobl 2005, 522f.). Im Austrofaschismus und Nationalsozialismus lebten kaum MuslimInnen im heutigen Gebiet von Österreich. Das führte in weiterer Folge dazu, dass das Islamgesetz erst in der Zweiten Republik nach der Einwanderung von muslimischen Studierenden aufgegriffen wurde und besonders nach der Einwanderung von so genannten Gastarbeitern wieder von Relevanz wurde. Folgt man dem Konzept der Pfadabhängigkeit, so würde diese die Genehmigung der IGGiÖ der Tradition der österreichischen Religionspolitik, welche eine korporatistische Hereinnahme von Kirchen und Religionsgesellschaften pflegt, erklären. Diese Inklusion bedeutet, dass der Staat privilegierte Kirchen und Religionsgesellschaften als Körperschaften des öffentlichen Rechts anerkennt. Mit diesen teilt er sich sogenannte "gemeinsame Angelegenheiten“. Im Gegensatz zu den „inneren Angelegenheiten“ einer Kirche oder Religions- gesellschaft, die in den autonomen Bereich dieser fällt, wird in "gemeinsamen Angelegenheiten" wie etwa im öffentlichen Bildungsbereich kooperiert, wie dies mit dem Religionsunterricht an öffentlichen Schulen der Fall ist, der inhaltlich von den jeweiligen Kirchen und Religionsgesellschaften verantwortet wird, während er staatlich finanziert wird. Diese Regelung geht auf Art. 15 StGG I867 zurück, die auch in der 2. Republik das Verhältnis von Staat und KuR bestimmte (Kalb 1994).

Tatsächlich lässt sich die übrige Religionspolitik zu dieser Zeit mit dem Verweis auf Pfadabhängigkeit erklären. Zwischen 1945 und 1979 wurden zwei Religionsgesellschaften gesetzlich anerkannt; 1972 die ArmenischApostolische Kirche in Österreich (BGBl. 1973 4/6) und I955 die Kirche Jesu Christi der Heiligen der Letzten Tage (Mormonen) (BGBl. Nr. 339/1955). Im Falle der Mormonen führte eine erste vom Verwaltungsgerichtshof aufgehobene Zurückweisung im Jahre 1953 zwei Jahre später zur Anerkennung derselben (Kalb/Potz/Schinkele 2003, 650). Beide Religionsgesellschaften waren quantitativ betrachtet vergleichbar klein wie jene der MuslimInnen. Zudem kam es I96I zu einer Novellierung des Protestantegesetzes, welches der Evangelischen Kirche A. und H.B. anlehnend an den völkerrechtlichen Vertrag mit dem Vatikan (Konkordat) eine Vielzahl an Privilegien einräumte (Kalb/Schinkele/Potz 2003). Dem Konzept der Pfadabhängigkeit folgend, müsste diese Religionspolitik ebenso im Zusammenhang mit dem Islam Anwendung gefunden haben, da ein jeder Schritt entlang eines Pfades einen jeden weiteren entlang diesem bekräftigt und gleichzeitig die Wahrscheinlichkeit des Abweichens von diesem Pfad durch Ergreifen einer alternativen Option einer politischen Handlung vermindert (Sorensen 2015, 2I). Selbst im Sinne eines vom soziologischen Institutionalismus beeinflussten Verständnisses von Pfadabhängigkeit (Thelen 1999, 386f.) würden die staatlichen Behörden der Tradition der österreichischen Religionspolitik folgen, indem sie das bereits vorhandene Sondergesetz für MuslimInnen (IslamG I9I2) administrativ umsetzen und entsprechend des ersten Artikels eine Religionsgesellschaft anerkennen. Es wäre nicht einmal eine völlig neue Institution zu schaffen gewesen, da das Gesetz bereits „den Anhängern des Islam [...] die Anerkennung als Religionsgesellschaft" (Art. I IslamG I912) zuerkennt. Es wäre lediglich entsprechend des Gesetzes gewesen, die Institution einer islamischen Religionsgesellschaft zu genehmigen, was mit der Pfadabhängigkeit des historischen Institutionalismus erklärbar gewesen wäre. Dies geschah jedoch nicht.

Der Muslimische Sozialdienst (MSD) nahm bereits in den 1960er Jahren das Ziel, eine solche Körperschaft zu gründen, in seine Statuten auf und fühlte im Unterrichtsministerium in dieser Angelegenheit vor (BMUK 1978). Auf ein erstes Ansuchen kam eine negative Antwort. Nach Informationen des Rechtsanwalts des MSD, 
Heinrich Orator, hieß es, den Behörden sei es „am sympathischsten [...], würde die Erfüllung von einzelnen seelsorgerischen Funktionen von einem nach dem Vereinsgesetz gebildeten Hilfsverein“ (zit. n. Balic 1995, 29) erfüllt werden. Nach Balic, dem ersten Vorsitzenden des MSD, würde „die Notwendigkeit der Klärung der bestehenden Riten und Schulen des Islams [...] das zuständige Ressortministerium umfangreiche Vorarbeiten kosten“" (ebd.). Zudem wurde die Subventionierung von ReligionslehrerInnen als weiteres Problem gesehen (ebd.). Die Empfehlung der Ausübung der religiösen Praxis auf Basis des Vereinsrechts bedeutet, dass die staatliche Behörde der religionsrechtlichen Praxis der Anerkennung als privilegierte Körperschaft öffentlichen Rechts nicht nachgekommen wollte. Diese Reaktion des Kultusamtes ist insofern bemerkenswert, als die Praxis der Vereinsbehörde bis in die beginnenden I980er Jahre jene war, dass Religionsgemeinschaften die Konstituierung als Verein ohnehin untersagt wurde. Dies wurde nur für Vereine, die religiöse Teilzwecke verfolgten und nicht das religiöse Leben der Mitglieder insgesamt zu erfassen trachteten, gewährt (Kalb/Potz/Schinkele 2003, I27f.). Damit hätte die Anerkennung als bloßer Verein, wie sie vom Kultusamt vorgeschlagen wurde, auch einer weiteren politischen Praxis widersprochen. Insofern kann deutlich gesagt werden, dass die zuständige staatliche Behörde alles andere als nach dem Prinzip der Pfadabhängigkeit gehandelt hatte.

Der Antragsuchende MSD hat nach Angaben eines Protokolls des Unterrichtsministeriums am 26. Jänner 197I erstmals formell ein Gesuch um Gründung einer islamischen Kultusgemeinde beim Bundesministerium für Unterricht und Kunst eingereicht (BMUK 19). Dieses wurde jedoch mehrere Male überarbeitet, bis es am 2. Mai 1979 letztendlich zu einer Genehmigung kam. Diese Genehmigung der Islamischen Glaubensgemeinschaft in Österreich basierte auf dem IslamG I9I2, die in ihrer Form entsprechend des österreichischen Religionsrechts gestaltet wurde. Bis es dazu kam, fanden zwischen 1975 und 1978 jeweils einmal jährlich vier Verhandlungen im Kultusamt des Bildungsministeriums, welches mit den gesetzlich anerkannten KuR betraut war, statt, so einer der damaligen Verhandler und spätere zweite Präsident der IGGiÖ, Anas Schakfeh (Schakfeh 2oria). Die Theorie des historischen Institutionalismus würde entsprechend dem Konzept der Pfadabhängigkeit kein IO-Jahre langes Warten nahelegen. Das Vorhaben wurde nicht grundlos so langsam entgegengenommen. Unterschiedliche Bedenken herrschten seitens der österreichischen Behörden, wie die Protokolle des Kultusamtes und des MSD zeigen.

Zum einen gab es finanzielle Bedenken, weil eine Anerkennung als Religionsgesellschaft verschiedene Rechte mit sich bringt. Die als am strittigsten vorgebrachte Argumentation war jene der Angst der öster- reichischen Behörden, männliche Muslime könnten ein Recht auf Polygamie einfordern, indem sie sich auf die Religionsfreiheit berufen (BMUK 1978). Das verwundert insofern, als bereits das IslamG $1912 \S 7$ dies explizit ausschließt, als das Eherecht noch konfessionell gebunden war (Potz/Schinkele 20I4). Dennoch wurde dieser Vorbehalt seitens der Antragsteller 1972 mit einem Gutachten der Türkischen Religionsbehörde (Diyanet 1972) und I976 mit einem Gutachten der ägyptischen Universität Al-Azhar entkräftet (Al-Azhar 1976). Das Begehren wurde aber nicht weiter entgegengenommen. Keine endogenen Faktoren kamen zum Tragen, die eine Kontinuität in der Religionspolitik hätten erkennen lassen. Es tauchten ebenso keine kritischen Entscheidungspunkte (critical junctures) auf, die eine Abweichung von der gewohnten Religionspolitik begründet hätten (Sorensen 2015, 2528). So könnte ganz im Gegenteil im Sinne des soziologischen Institutionalismus argumentiert werden, dass die Bedenken gegenüber dem Islam und seinem klassischen Familienbild Skepsis auf Seiten der Behörden hervorbrachten.

Wenn die handelnden Akteure nicht nach dem Prinzip der Pfadabhängigkeit gehandelt hatten, warum wurde die IGGiÖ schließlich doch im Jahre I979 anerkannt und damit das IslamG 1912 umgesetzt? Warum wurden all die Bedenken, die es gab, schlussendlich hintangestellt? Zur Schließung dieser Erklärungslücke, der in der Literatur nicht nachgegangen wird, wird die Theorie der politischen Möglichkeitsstrukturen (political opportunity structures) herangezogen, wonach externe Faktoren bestimmte Politiken erst ermöglichen. Es wird danach gefragt, ob es bestimmte politische Möglichkeitsstrukturen waren, die schließlich 1979 doch noch zu einer Errichtung einer islamischen Religionsgesellschaft führten.

\section{Politische Möglichkeitsstrukturen: Österreichs Kulturdiplomatie}

Die interministeriellen Protokolle zeigen, dass Österreichs Rolle in der internationalen Politik eine Gelegenheitsstruktur bildete. Ein Schreiben des damaligen zuständigen Ministerialbeamten verweist darauf, dass das Ansuchen des MSD auf Ordnung der äußeren Rechtsverhältnisse vorerst deswegen zögerlich aufgenommen wurde, weil das Projekt zur Errichtung eines Islamischen Zentrums in Wien keine Aktivitäten entfaltet hatte.

Das Islamische Zentrum Wien war eine von verschiedenen islamischen Staaten gegründete Stiftung, die unter Vorsitz des ägyptischen Botschafters 1967 einen Stiftungsbrief im Unterrichtsministerium unterzeichnete, worauf ein Jahr später eine feierliche Grundsteinlegung unter Anwesenheit des damaligen Außenministers Kurt 
Waldheim, Kardinal Franz König sowie von Botschaftern vieler islamischer Länder geschah (Hadžić 20I3, 97). Nachdem jedoch der Botschafter Ägyptens abberufen worden war, entfalteten das Zentrum keine weiteren Aktivitäten, womit im Kultusamt argumentiert wurde, das Ansuchen des MSD auf Gründung einer islamischen Religionsgesellschaft positiv zu beantworten (BMUK I978). Aus Geldmangel begann der Baubeginn erst ab dem I. Juli 1977. Es scheint, als wäre vonseiten der Regierung eine zentrale Überlegung jene gewesen, mit der Errichtung des Islamischen Zentrums Wien oder mit Errichtung der IGGiÖ das internationale Ansehen Österreichs - besonders in der islamischen und arabischen Welt - zu steigern. Darauf weist nicht nur die Anwesenheit des Außenministers anstatt des Bundeskanzlers bei der Unterzeichnung des Stiftungsbriefes. In einem Schreiben vom damaligen Verfassungsrechtler des Bundeskanzleramtes, Ludwig Adamovich, heißt es am 6. April 1979 bezugnehmend auf das Ersuchen des Kultusamtes auf Anerkennung der IGGiÖ:

„Das Bundeskanzleramt-Verfassungsdienst vertritt die Auffassung, dass auch der Aspekt in die Überlegungen mit einbezogen werden sollte, dass eine Novellierung oder Neuerlassung des Islamgesetzes im Hinblick auf die zunehmende Anzahl der in Wien beschäftigten Diplomaten aus islamischen Ländern zweckmäßig wäre (z.B. UNO, OPEC)“ (Adamovich 1979, 2).

Damit wird deutlich, dass der Versuch, Österreich im internationalen Kontext als offen gegenüber der islamischen Religion zu positionieren, Teil der Strategie des Bundeskanzleramtes war. Dies zeigt sich etwa daran, dass bei der Eröffnung des islamischen Zentrums neben Bundeskanzler Kreisky und Bundespräsident Kirchschläger, auch Außenminister Pahr, sowie Vertreter verschiedener muslimischer Länder anwesend waren (Hadžić 2OI3, 98). Mit dem Verweis Adamovichs kann die Anerkennung der IGGiÖ als Puzzleteil in der Positionierung des damaligen Bundeskanzlers Bruno Kreisky gesehen werden, was insbesondere die Dimension der Machtasymmetrie veranschaulicht. Kreiskys ,aktive Neutralitätspolitik' führte ihn als Gesandten der Sozialistischen Internationale im März 1974 zu Sadat und Nasser wie auch zu Gaddafi und Arafat, der damals noch weithin als Terrorist gehandhabt wurde. Kreisky war für seine pro-palästinensische Haltung bekannt. Er bot der Palästinensischen Befreiungsorganisation, PLO, im Jahre 1980 einen Sitz für einen Botschaftsvertreter in Wien an, als diese noch von einer Marginalisierung und Ausgrenzung durch die internationale Gemeinschaft betroffen war (Kriechbaum 2004, 26I-283). Damit konnte sich Kreisky für Österreich Anerkennung in der muslimischen Welt verschaffen. Das zeigt sich auch daran, dass in späteren Jahren die Führung der IGGiÖ immer wieder im Rahmen von Staatsbesuchen Teil der österreichischen Delegation war (Schakfeh 20Irb).

Diese Dimension der internationalen Politik bekundete Bundespräsident Heinz Fischer später in einer Rede am 2I. Oktober 2008, als dieser Anas Schakfeh das Große Goldene Ehrenzeichen mit dem Stern für Verdienste um die Republik verlieh. In seiner Rede meinte er:

\section{„Ihre internationalen Initiativen, sehr geehrter Herr Präsident, hatten weit über Österreich hinaus positi- ve Wirkungen für das Zusammenleben der Muslime in verschiedenen europäischen Staaten, für gute Di- aloge zwischen Muslimen und Nichtmuslimen und für das Ansehen unseres Landes in der internationa- len Staatengemeinschaft" (Fischer 2008).}

,Weiche Macht' (soft power) (Nye 2004) bzw. Kulturdiplomatie ist meist eine Begleiterscheinung, harter Macht', in diesem Fall wirtschaftlicher Macht. Während der unerwartete Öl-Schock im Jahre 1973 Österreich unerwartet traf, konnte die Herausforderungen mit austro-keynesianischen Methoden noch aufgefangen werden. Die zweite Ölkrise in den Jahren 1978-1979 traf Österreich hingegen um einiges härter. Die Nationalbank verlor beinahe ein Drittel ihrer Reserven und der Zins musste deutlich erhöht werden (Unger/Heitzmann 2003, 375f.). Bereits nach der ersten Ölpreiskrise suchte Kreisky mit seinen Verbindungen zur arabisch-islamischen Welt die wirtschaftlichen Interessen seines kleinen Landes zu sichern. Die Staatsausgaben stiegen ab I974 auf über 48\% im Jahr 1978 an (Getzner/Neck 200I). Die österreichische Industrie unterstützte dieses Vorhaben, weil sie davon profitierte in industriell weniger entwickelten Ländern Produkte zu verkaufen, die wiederum mit günstigen Ressourcen die österreichische Wirtschaft entlasten konnten. Tatsächlich - wenn auch nicht nur deswegen - gelang es der Industrie trotz Rezession, bis Anfang der 1980er Jahre den Wachstumsvorsprung gegenüber den restlichen europäischen OECD-Ländern aufrecht zu halten (Volk/Wieser 1986, 27). Das hatte mitunter mit der offensiven Positionierung österreichischer Unternehmen im arabischen Raum zu tun (Horvath 2009, IO2). Dass Österreich Vorreiter in westlich-arabischen Beziehungen war, darauf deutet etwa dass die Austrian Airlines als erste europäische Fluglinie arabische Destinationen anflog (Woditschka 20I5). Der explizite Verweis Adamovichs auf Diplomaten aus muslimischen Ländern, die in der UNO und OPEC angestellt sind, veranschaulicht die Bedeutung, welche die österreichische Politik dazumal österreichisch-arabischen Beziehungen beimaß. Dies wurde nicht zuletzt durch die Eröffnung des Vienna International Center im August 1979 gestärkt, welche Wien zur dritten UNO-Stadt machte (Petritsch 20I0, 29If.). Die Dimension, weicher Macht' in der 
Inklusion des Islams in das religionsrechtliche System erhält hier ihre Logik.

Somit kann gesagt werden, dass die Anerkennung der IGGiÖ im Jahre I979 auf Basis des Islamgesetzes aus dem Jahre I9I2 durch die Rolle Österreichs in der internationalen Politik sowie auf Grundlage wirtschaftlicher Kalküle als politische Möglichkeitsstrukturen forciert wurde. Eine Anerkennung des Islams in Österreich als eines der wenigen Länder Westeuropas (Potz/Wieshaider 2004) sollte die politische Offenheit Österreichs demonstrieren. Folgt man der Aussage Adamovichs, würden die in Österreich ansässigen Diplomaten aus der muslimischen Welt die Anerkennung zur Kenntnis nehmen und damit Österreich zu noch mehr Ansehen in der muslimischen Welt verhelfen. Den österreichischen Behörden standen mehrere Optionen im Umgang mit dem Antrag seitens des MSD offen. Das zögerliche Handeln der Beamten und insbesondere die - historisch eingeschriebene - Sicht auf den Islam als kulturell ,Anderes' (Gingrich 2015), wie es sich im Hinblick auf die Befürchtung um Polygamie zeigt, hätte eine Beendigung der Pfadentwicklung in der österreichischen Religionspolitik bedeuten können. Aus der Perspektive der Politischen Möglichkeitsstrukturen kann deshalb gesagt werden, dass die kulturdiplomatische Dimension des Islamgesetzes ein Möglichkeitsfenster bot, die zur de facto-Anerkennung des Islams in der Zweiten Republik geführt hat. Damit kann festgehalten werden, dass eine vertiefte Institutionalisierung des Islams erst in der Zweiten Republik geschah. Die religionsrechtliche Tradition Österreichs wurde damit erst ab I979 wirksam.

\section{Zusammenfassung und Ausblick}

In diesem Artikel konnte gezeigt werden, dass die korporatistische Hereinnahme des Islams in der Zweiten Republik im Jahre 1979, die zur Genehmigung der IGGiÖ führte, im Wesentlichen mit beiden Theorien, jener der Politischen Möglichkeitsstrukturen sowie jener des historischen Institutionalismus, erklärt werden kann. Während der historische Institutionalismus und sein Konzept der Pfadabhängigkeit die Form der Anerkennung entsprechend der historisch-gewachsenen Staats-Kirche-Beziehungen erklären können, liefert der historische Institutionalismus keine Erklärung für das warum. Eine Anerkennung der IGGiÖ als Körperschaft öffentlichen Rechts in der Zweiten Republik war erst durch eine aktive Zustimmung der politischen AkteurInnen möglich. MuslimInnen hätten sich wie in Deutschland auch lediglich auf Basis des Vereinsrechts organisieren können, wo ein ähnliches Staats-KircheVerhältnis existiert. Dies war auch der ursprünglich erste Vorschlag seitens der staatlichen Behörden gegenüber den um Genehmigung ansuchenden MuslimInnen. $\mathrm{Zu}$ - dem zeigte der Prozess des Anerkennungsverfahrens, dass dieser mit Friktionen bestückt war und sich im Gegensatz zu anderen religionsrechtlichen Anerkennungen wie gegenüber den Armeniern und den Mormonen oder der Aufwertung der Protestantischen Kirche über einen Zeitraum von mehr als zehn Jahren zog. Die Angst um die Polygamie-Frage veranschaulicht, dass es nicht lediglich ein behördlicher Akt war, diese Anerkennung auszusprechen und gleichzeitig Ängste bestanden.

Anhand der Analyse von behördlichen Dokumenten, Reden und Interviews konnte gezeigt werden, dass die korporatistische Inklusion des Islams als Möglichkeitsfenster für ein positives Image und eine stärkere internationale Rolle Österreichs im Nahen Osten und damit einhergehend zur Erlangung wirtschaftlicher Interessen eine Überlegung darstellte. Diese kulturdiplomatische Dimension wurde auch in späteren Jahren bewusst eingesetzt, wie das Handeln der politischen Eliten (der ÖVP) zeigt, die das „Sondermodell Österreich“ Anfang der 20ooer Jahre gepriesen und als gelungenes Beispiel eines Gegennarrativs zur Theorie des Kampfes der Kulturen präsentierte (Sticker 2008).

Die inklusive korporatistische Hereinnahme des Islams in das politische System und die damit einhergehende - oft mit dem Adjektiv, liberal' und ,tolerant' versehene - Islampolitik bedarf vermehrter kritischer Würdigungen. Die Katholische Kirche hat mit dem völkerrechtlich geschlossenen Vertrag, dem Konkordat, eine privilegierte Position, mit der andere anerkannte Kirchen und Religionsgesellschaften keineswegs auf $\mathrm{Au}$ genhöhe stehen. Im Zusammenhang mit der Ausübung von Religionsfreiheit zeigen weitere Untersuchungen im Zusammenhang mit Moscheebauten wie etwa jene von Fürlinger (2OI3) und mir (2OIO), dass die Religionsfreiheit von MuslimInnen in Österreich mit staatlichen, gesellschaftlichen und politischen Praxen eingeschränkt werden kann. Weitere Untersuchungen sollten sich basierend auf Arbeiten über den diskursiven Paradigmenwechsel im Zusammenhang mit dem Islamthema (Hafez 2015) und zum neuen Islamgesetz 2015, das vielseitiger Kritik ausgesetzt war, mit der Frage auseinandersetzen, inwiefern das neue Islamgesetz eine Kontinuität bzw. eine Wende in der Islampolitik der derzeitigen und kommenden Regierungen der Zweiten Republik bedeutet und welche Erklärungsmodelle diese Politiken nachvollziehbar machen. Es sollte in diesem Zusammenhang auch gefragt werden, inwiefern der historische Institutionalismus und sein Konzept der Pfadabhängigkeit die Islampolitik der Zweiten Republik erklären können. 


\section{Literatur}

Adamovich, Ludwig (1979). Brief betreffend Anerkennung einer islamischen Religionsgemeinde in Wien, GZ 602212/I-VI/5/79, 6. April 1979.

Bennett, Andrew/Alexander L. George (2005). Case Studies and Theory Development in the Social Sciences, London.

Berghahn, Sabine/Petra Rostock (Hg.) (2009). Der Stoff aus dem Konflikte sind. Debatten um das Kopftuch in Deutschland, Österreich und der Schweiz, Bielefeld.

Al-Azhar (1976). Brief des Großimams der Al-Azhar, Abdal-Halim Mahmud an Islamil Balic, Präsident der Vereinigung der österreichischen Muslime, I3. Februar 1976.

Balic, Smail (1995). Zur Geschichte der Muslime in Österreich, in: Susanne Heine (Hg.): Islam zwischen Selbstbild und Klischee. Eine Religion im österreichischen Schulbuch, Wien, 23-36.

Bauböck, Rainer/Bernhard Perchinig (2006). Migrationsund Integrationspolitik, in: Herbert Dachs/Peter Gerlich/Herbert Gottweis/Helmut Kramer/Volkmar Lauber/Wolfgang C. Müller/Emmerich Tálos (Hg.): Politik in Österreich, Wien, 726-743.

Beyer, Jürgen (2006). Pfadabhängigkeit: Über institutionelle Kontinuität, anfällige Stabilität und fundamentalen Wandel, Schriften aus dem Max-Planck-Institut für Gesellschaftsforschung, Bd. 56, Frankfurt a.M.

$B G B l$. Nr. 339/1955, Verordnung des Bundesministeriums für Unterricht vom 27. September 1955, betreffend die Anerkennung der Anhänger des Religionsbekenntnisses der "Kirche Jesu Christi der Heiligen der Letzten Tage" (Mormonen) als Religionsgesellschaft.

BGBl. 1973 4/6, Verordnung: Anerkennung der Anhänger der Armenisch-apostolischen Kirche in Österreich als Religionsgesellschaft.

$B M U K$ (1978). Geschäftszahl 9076/I-9c/78.

De Chilla, Rudolf/Martin Reisigl/Ruth Wodak (1998). Zur diskursiven Konstruktion nationaler Identität, Frankfurt a.M.

Diyanet (1972). Din İs.Y.Krl.Bşk.D/I-3/72, I8 Dezember 1972.

Dolezal, Martin/Marc Helbling/Swen Hutter (2010). Debating Islam in Austria, Germany and Switzerland: Ethnic Citizenship, Church-State Relations and Right-Wing Populism, West European Politics, Vol. 33(2), I7I-I9O.

Fetzer, Joel S./J. Christopher Soper (2005). Muslims and the State in Britain, France, and Germany, Cambridge.

Fischer, Heinz (2008). Rede: Überreichung des Großen Goldenen Ehrenzeichens mit dem Stern an den Präsidenten der Islamischen Glaubensgemeinschaft in Österreich, Professor Anas Schakfeh, 21. IO. 2008, Internet: http://www.bundespraesident.at/newsdetail/ artikel/ueberreichung-des-grossen-goldenen-ehrenzeichens-mit-dem-stern-an-den-praesidentender-islamischen-g/ (26.03.2016)

Furat, Ayse Zisan (2012). A Cultural Transformation Project: Religious and Educational Policy of the Austro-Hungarian Empire in Bosnia (I878-I9I8), in: Er, Hamit/k/Er/k/Ayse Zisan Furat (Hg.): Balkans and Islam: Encounter, Transformation, Discontinuity, Continuity, Newcastle, 63-84.

Fürlinger, Ernst (2013). Moscheebaukonflikte in Österreich. Nationale Politik des religiösen Raums im globalen Zeitalter, Wien.

Horvath, Elisabeth (2009). Heinz Fischer. Die Biografie, Wien.

Goodwin, Jeff/James M. Jasper (1999). Caught in a Vinding, Snarling Vine: The Structural Bias of Political Process Theory, in: Sociological Forum, Vol. I4(I), 27-92.

Gresch, Nora/Leila Hadj-Abdou/Sieglinde Rosenberger/Birgit Sauer (2008). Tu felix Austria?: The Headscarf and the Politics of ,Non-issues', in: Social Politics: International Studies in Gender, State and Society, Vol. I5(4), 4II-432.

Gresch, Nora/Leila Hadj-Abdou/Sieglinde Rosenberger/ Birgit Sauer (2012), Hijabophobia revisited: Kopftuchdebatten und -politiken in Europa. Ein Überblick über das Forschungsprojekt VEIL, in: Eva Hausbacher/Elisabeth Klaus/Ralph Poole/Ulrike Brandl/Ingrid Schmutzhart (Hg.): Migration und Geschlechterverhältnisse. Kann die Migrantin sprechen?, Wiesbaden, 198-212.

Gringrich, Andre (2015). The Nearby Frontier: Structural Analyses of Myths of Orientalism, in: Diogenes, Vol. $60(2), 60-66$.

Gruber, Helmut (1991). Antisemitismus im Mediendiskurs: „Die Affäre Waldheim“ in der Tagespresse, Wiesbaden.

Getzner, Michael/Reinhard Neck (200I). Die Entwicklung der Staatsausgaben in Österreich. Ludwig Boltzmann-Institut zur Analyse Wirtschaftspolitischer Aktivitäten, Wien.

Hafez, Farid (2010). Islamophober Populismus: Moscheeund Minarettbauverbote österreichischer Parlamentsparteien, Wiesbaden.

Hafez, Farid (20I4). Gedenken im „islamischen Gedankenjahr". Zur diskursiven Konstruktion des österreichischen Islams im Rahmen der Jubiläumsfeier zu IOo Jahren Islamgesetz, in: Wiener Zeitschrift für die Kunde des Morgenlandes, 63-85.

Hafez, Farid (2015). Das IslamG im Kontext islamophober Diskurse. Eine Policy Frame-Analyse zum Politikgestaltungsprozess des IslamG 2015, juridikum 2/2015, I60-I65.

Hall, Peter A./Rosemary C. R. Taylor (1996). Political Science and the Three New Institutionalisms, in: Political Studies, Vol. 44(5), 936-57. 
Hanisch, Ernst (1977). Die Ideologie des politischen Katholizismus in Österreich 19I8-I938. Vol. 5. Geyer.

Hanisch, Ernst (1994). Der lange Schatten des Staates: Österreichische Gesellschaftsgeschichte im 20. Jahrhundert, I890-1990.

Helms, Ludger (2004). Einleitung: Politikwissenschafltiche Institutionenforschung am Schnittpunkt von Politischer Theorie und Regierungslehre, in: Ludger Helms/Uwe Jun (Hg.): Politische Theorie und Regierungslehre. Eine Einführung in die politikwissenschaftliche Institutionenforschung, Frankfurt a.M., 13-44.

Hödl, Klaus (20I0). Islamophobia in Austria: the Recent Emergence of Anti-Muslim Sentiments in the Country in: Journal of Muslim Minority Affairs, Vol. $30(4), 443-456$.

Hadžić, Halima (2013). Die kommunikativen Leistungen des Moslemischen Sozialdienstes als Träger des religiösen und sozialkulturellen Lebens der Muslime in Österreich: von 1962-1979, Wien.

Kalb, Herbert (1994). „Stufenweise Anerkennung.“ Einige Überlegungen zum Anerkennungsgesetz, in: Herbert Schambeck/Hans Walther Kaluza (Hg.): Recht, Glaube, Staat: Festgabe für Herbert Schambeck, Berlin, I99-2I4.

Kalb, Herbert/Richard Potz/Brigitte Schinkele (2003). Religionsrecht, Wien.

Kingdon, John W. (1984). Agendas, Alternatives, and Public Policies, Boston.

Krasner, Stephen D. (1984). Approaches to the State: Alternative Conceptions and Historical Dynamics, in: Comparative Politics, Vol. I6(2), 223-246.

Krzyzanowski, Michal/Ruth Wodak (20II). The Politics of Exclusion: Debating Migration in Austria, New Brunswick, NJ.

Meyer, David S./Debra C. Minkoff (2004). Conceptualizing Political Opportunity, in: Social forces, Vol. 82(4), I457-I492.

McAdam, Doug (1996). Conceptual Origins, Problems, Future Directions, in: Doug McAdam/John D. McCarthy/Zald N. Mayer (Hg.): Comparative Perspectives on Social Movements: Political Opportunties, Mobilizing Structures, and Cultural Framings, Cambridge, 23-40.

Muno, Wolfgang (2009). Fallstudien und die vergleichende Methode, in: Gert Pickel/Hans-Joachim Lauth/Detlef Jahn (Hg.): Methoden der vergleichenden Politikund Sozialwissenschaft, Wiesbaden, II3-I32.

Nye, Joseph Jr. (2004). Soft Power. The Means to Success in World Politics, New York.

Petritsch, Wolfgang (20I0). Bruno Kreisky. Die Biografie, Wien.

Potz, Richard (2013). Das Islamgesetz I9I2 - eine österreichische Besonderheit, SIAKJournal - Zeitschrift für Polizeiwissenschaft und polizeiliche Praxis, Vol. I, $45-54$.

Potz, Richard/Wolfgang Wieshaider (Hg.) (2004). Islam and the European Union, Leuven.

Potz, Richard/Brigitte Schinkele (20I4). Stellungnahme zum Entwurf eines Bundesgesetzes, mit dem das Gesetz betreffend die Anerkennung der Anhänger des Islam als Religionsgesellschaft geändert wird (69/ME XXV. GP) 5. November 2014.

Pelinka, Anton/Hubert Sickinger/Karin Stögner (2008). Kreisky - Haider. Bruchlinien österreichischer Identitäten, Wien.

Pelinka, Anton/Ruth Wodak (Hg.) (2002). „Dreck am Stecken": Politik der Ausgrenzung, Wien.

Peters, Guy B. (1999). Institutional Theory in Political Science. The 'New Institutionalism', London.

Peters, B. Guy/Jon Pierre/Desmond S. King (2005). The Politics of Path Dependency: Political Conflict in Historical Institutionalism, in: The Journal of Politics, Vol. 67(4), I275-I300.

Prainsack, Barbara (2006). Religion und Politik, in: Herbert Dachs/Peter Gerlich/Herbert Gottweis/Helmut Kramer/Volkmar Lauber/Wolfgang C. Müller/Emmerich Tálos (Hg.): Politik in Österreich, Wien, 538-549.

Reiterer, Albert F. (1996). Kärntner Slowenen: Minderheit oder Elite? Neuere Tendenzen der ethnischen Arbeitsteilung, Klagenfurt.

Rosenberger, Sieglinde/Julia Mourao Permoser (2012). Religiöse Staatsbürgerschaft: Widersprüche der Governance von Diversität, in: Kurt Appel/Christian Danz/ Richard Potz/Sieglinde Rosenberger/Angelika Walser (Hg.): Religion in Europa heute. Sozialwissenschaftliche, rechtswissenschaftliche und hermeneutischreligionsphilosophische Perspektiven, Wien, 67-84.

Schakfeh, Anas (201ra). Interview des Autors mit Anas Schakfeh, 7 Juli 20 II.

Schakfeh, Anas (20Irb). Interview des Autors mit Anas Schakfeh, I3 Juli 20 II.

Sticker, Maja (2008). Sondermodell Österreich? Die Islamische Glaubensgemeinschaft in Österreich, Klagenfurt.

Sorensen, Andre (2015). Taking Path Dependence Seriously: an Historical Institutionalist Research Agenda in Planning History, in: Planning Perspectives, Vol. 3O(I), I7-38.

Spielhaus, Riem/Martin Herzog (2015). Die rechtliche Anerkennung des Islams in Deutschland. Ein Gutachten für die Friedrich-Ebert-Stiftung, Arbeitspapier Religion und Politik, Berlin.

Strobl, Anna (2005). Der österreichische Islam. Entwicklung, Tendenz und Möglichkeiten, SWS-Rundschau, Vol. 45(4), 520-543.

Thelen, Kathleen (1999). Historical Institutionalism in Comparative Politics, Annual Review of Political Science, Vol. 2, 369-404. 
Unger, Brigitte/Karin Heitzmann (2003). The Adjustment Path of the Austrian Welfare State: Back to Bismarck? in: Journal of European Social Policy, Vol. I3(4), 37 I-387.

Volk, Ewald/T. Wieser (1986). Industriepolitik in der Krise?, Die Entwicklung der österreichischen Industriepolitik und ihrer Bestimmungsgründe seit I945, Gutachten im Auftrag des Bundesministeriums für Wissenschaft und Forschung, Wien.

Wandruszka, Adam (1985). Katholisches Kaisertum und multikonfessionelles Reich, in: Adam Wandruszka (Hg.): Die Habsburgermonarchie I848-I9I8, Band 4: Die Konfessionen, Wien: VÖAW.

Woditschka, Elisabeth (2015). Der Betriebsrat als Showstar, in: Forbes Austria, Juli 20I5, 44-45.

\section{Autor}

Farid Hafez ist wissenschaftlicher Mitarbeiter an der Abteilung Politikwissenschaft der Universität Salzburg. Forschungsaufenthalte in den USA (Berkeley, Columbia), Lehraufenthalte in Indonesien, der Türkei und den USA. Seit 20 Io ist er Herausgeber des bilingualen Islamophobia Studies Yearbook, seit 2016 Mitherausgeber des European Islamophobia Report. 2009 erhielt er gemeinsam mit John Bunzl den Bruno-Kreisky-Anerkennungspreis für „Islamophobie in Österreich“ für das politische Buch des Jahres. Zuletzt erschienen in zweiter Auflage: „Islamisch-politische Denker. Eine Einführung in die islamisch-politische Ideengeschichte" (Peter-Lang Verlag). Zahlreiche Publikationen zu Rassismus, Islam, Rechtspopulismus. Forschungsschwerpunkte: StaatsKirche-Beziehungen, Jugendkultur und soziale Bewegungen, Rassismus. 
